\title{
Medium Optimization by Response Surface Methodology for Improved Cholesterol Oxidase Production by a Newly Isolated Streptomyces rochei NAM-19 Strain
}

\author{
Elsayed Ahmed Elsayed (iD) ${ }^{1,2}$ and Nayera Ahmed Abdelwahed ${ }^{2}$ \\ ${ }^{1}$ Bioproducts Research Chair, Zoology Department, College of Science, King Saud University, Riyadh 11451, Saudi Arabia \\ ${ }^{2}$ Department of Chemistry of Natural and Microbial Products, National Research Centre, Dokki, Cairo 12622, Egypt \\ Correspondence should be addressed to Elsayed Ahmed Elsayed; eaelsayed@ksu.edu.sa
}

Received 5 November 2019; Revised 1 January 2020; Accepted 8 January 2020; Published 31 March 2020

Academic Editor: Yeo J. Yoon

Copyright (c) 2020 Elsayed Ahmed Elsayed and Nayera Ahmed Abdelwahed. This is an open access article distributed under the Creative Commons Attribution License, which permits unrestricted use, distribution, and reproduction in any medium, provided the original work is properly cited.

\begin{abstract}
Cholesterol oxidase is an alcohol oxidoreductase flavoprotein with wide biotechnological applications. The current work describes the isolation of a potential cholesterol oxidase producing streptomycete from Egyptian soil. The isolated strain produced cholesterol oxidase in submerged culture using a medium containing glucose, yeast extract, malt extract, and $\mathrm{CaCO}_{3}$ with the addition of cholesterol as an inducer. The isolated strain was identified as Streptomyces rochei NAM-19 based on 16S rRNA sequencing and phylogeny. Optimization of cholesterol oxidase production has been carried out using response surface methodology. The Plackett-Burman design method was used to evaluate the significant components of the production medium followed by Box-Behnken experimental design to locate the true optimal concentrations, which are significantly affecting enzyme production. Results showed that the predicted enzyme response could be closely correlated with the experimentally obtained production. Furthermore, the applied optimization strategy increased volumetric enzyme production by 2.55 times $(65.1 \mathrm{U} / \mathrm{mL})$ the initial production obtained before medium optimization $(25.5 \mathrm{U} / \mathrm{mL})$.
\end{abstract}

\section{Introduction}

Cholesterol oxidase, CO (EC. 1.1.3.6) enzyme is a monomeric oxidoreductase flavoenzyme, which catalyzes the oxidation of cholesterol to cholesterone and hydrogen peroxide. Cholesterol oxidase is an industrially important enzyme, which is used, in combination with related enzymes, in the clinical determination of serum cholesterol levels [1]. In addition, the CO enzyme is used in the biological processes involving conversion of different steroidal and nonsteroidal compounds [2]. Moreover, CO has been used as an insecticide in transgenic crop-pest management [3]. Meanwhile, many attempts have been made to reduce the cholesterol content of foods by the $\mathrm{CO}$ enzyme [4]. CO enzyme biosensors have found applications in the detection of cholesterol level in various samples [5] and have also scientific importance in investigating cell membrane interactions with cholesterol [6]. Due to its vast range of applications, the industrial importance and demand of $\mathrm{CO}$ have gained increased interest. CO enzyme has been produced by different microorganisms in submerged cultures, e.g., Arthrobacter sp., Pseudomonas sp., Rhodococcus sp., Mycobacterium sp., Streptomyces sp., Nocardia, and Streptoverticillium [7, 8]. Furthermore, some pathogenic bacteria were found to produce $\mathrm{CO}$ as a part of their mechanism to interfere with cell membrane integrity, hence enabling them to invade the host cells [9]. Accordingly, since CO enzyme has many applications in food and medical sectors, therefore, it is of great importance that the producing organisms should have the GRAS (generally regarded as safe) status [10]. Accordingly, search for new and safe microorganisms capable of producing such an industrially important enzyme has continued. Streptomyces spp. have been known 
as the best producers of $\mathrm{CO}$ with the least possible virulence or pathogenicity [11].

The commercial production of microbial valued products is generally performed in submerged cultivation systems [12-14]. The optimization of the production process depends mainly on two strategies to improve the volumetric production capabilities of the producing strain; (i) optimization of medium composition and (ii) improvement of the production process parameters [15]. The optimization of the composition of the production medium is generally achieved by optimization of the medium composition either by OFAT (one-factor-at-a-time) or by statistical optimization approaches [16]. Although OFAT optimization is traditional and simple to use, it is time and cost consuming and requires many experiments to be finalized [17]. Moreover, OFAT optimization does not consider the interaction between different factors investigated [18]. On the other hand, statistical medium optimization approaches have the advantages of enabling nutrient rebalancing and enrichment of essential medium components, while reducing unnecessary ones; i.e., they allow investigating the interaction between different factors [19]. Additionally, statistical approaches reduce the number of experiments to be performed, which is greatly reflected in the costs and thus the economy of the production process [16]. Generally, the statistical optimization of variable medium components produces a definite optimal response, either cell growth or volumetric production, which can be obtained through factorial design and the use of response surface methodology (RSM). RSM is a powerful mathematical tool for testing multiple process variables, which reduces experimental trials required to evaluate the significant components affecting the microbial process $[20,21]$.

The aim of the current investigation was to isolate and molecularly identify a potential actinomycete strain capable of producing CO. Furthermore, the statistical optimization design was adopted to optimize the composition of the production medium through application of the RSM approach. This was achieved by implementing both PlackettBurman (PBD) and Box-Behnken (BBD) design methodologies. Finally, both unoptimized and statistically optimized medium compositions were compared in terms of total $\mathrm{CO}$ volumetric production.

\section{Materials and Methods}

2.1. Sample Collection and Isolation of Cholesterol Oxidase Producing Strains. Firstly, CO enzyme-producing microbial strains were isolated from different soil samples collected from El-Giza Governorate, Giza, Egypt. The isolation medium is composed mainly of mineral agar screening medium supplemented with cholesterol as the sole carbon source for growth. Serial dilutions were prepared from soil samples, from which loops were inoculated on the initial screening agar plates. CO-producing microbial colonies growing on the plate's surface appeared white and chalky with a characteristic soil smell, indicating cholesterol oxidization by the growing isolates. The potential of $\mathrm{CO}$ production was evaluated as follows: a loop full slant culture was suspended in sterile $0.85 \% \mathrm{NaCl}$ solution. A portion of the suspension was spread on cholesterol enrichment medium containing the following $(\mathrm{g} / \mathrm{L})$ : yeast extract, $10 ; \mathrm{KH}_{2} \mathrm{PO}_{4}, 0.05 ; \mathrm{NaNO}_{3}$, 1; $\mathrm{MgSO}_{4}, 0.5$; cholesterol (dissolved in 1\% Triton X-100), 2; and agar, 15. Plates were incubated at $32^{\circ} \mathrm{C}$ for 7 days. Colonies forming halos were streaked on $\mathrm{CO}$ indicator agar plates and incubated at $32^{\circ} \mathrm{C}$ for 2-4 days [11]. The development of intense brown pigmentation around the growing colonies indicates the production of $\mathrm{CO}$. These colonies were selected and maintained on slants containing starch nitrate agar medium composed of the following $(\mathrm{g} / \mathrm{L})$ : starch 20; $\mathrm{KNO}_{3}, 2 ; \mathrm{K}_{2} \mathrm{HPO}_{4}, 1 ; \mathrm{MgSO}_{4} .7 \mathrm{H}_{2} \mathrm{O}, 0.5$; and agar, 20. After sporulation, spores were suspended in $20 \%(\mathrm{v} / \mathrm{v})$ glycerol and were collected and stored at $-20^{\circ} \mathrm{C}$ for subsequent experiments.

2.2. Identification of CO-Producing Isolate. The most potent actinomycete isolates were grown for 7 days on starch agar slants at $32^{\circ} \mathrm{C} .2 \mathrm{ml}$ of a spore suspension was inoculated into starch nitrate broth and incubated for 3 days on an incubator shaker (Innova 4080, New Brunswick Scientific, NJ, USA) at $200 \mathrm{rpm}$ and $32^{\circ} \mathrm{C}$ to obtain heavy growth of vegetative cells (presporulation). The preparation of total genomic DNA was conducted in accordance with the methods described by Sambrook et al. [22]. PCR amplification of the 16S rRNA gene of the local actinomycete strain was conducted according to Edwards et al. [23]. Primers used in PCR amplification were the F27 primer (5-AGAGTTTGATCMTGGCTCAG-3) and the R1492 primer (5TACGGGYTACCTTGTTACGACTT-3). Purification and sequencing of PCR products of the isolate were performed and DNA sequence similarity was compared with sequences deposited in the Gene Bank database using the BLAST program (http://www.ncbi.nlm.nih.gov). Multiple sequence alignment and molecular phylogeny were evaluated using BLAST software [24]. Spore morphology and spore chain were investigated by Scanning Electron Microscopy (SEM) (JEOL, JSM-5910, Japan). A plug of agar containing the culture was removed and fixed in glutaraldehyde vapor $(2 \%$ v/v) at room temperature for 3 hours. Then, samples were dehydrated with increasing ethanol concentrations $(50,60$, 70,80 , and $95 \%$ ) for $15 \mathrm{~min}$ each and then twice with $100 \%$ ethanol (30 $\mathrm{min} /$ round). Ethanol was substituted with acetone and subjected to a critical point dryer (CPD7510, Polaron, Rang). The samples were sputter-coated with gold in an SPI-Module TM Sputter Coater (SPI Supplies, Division of Structure Probe Inc., USA) and observed by SEM.

2.3. Inoculum Preparation. Erlenmeyer flasks (250 mL) containing $100 \mathrm{~mL}$ of broth liquid medium were used to prepare the inoculum. The medium composed of the following $(\mathrm{g} / \mathrm{L})$ : glucose, 20; yeast extract, $10 ; \mathrm{KH}_{2} \mathrm{PO}_{4}, 0.05$; $\mathrm{NaNO}_{3}, 1$; and $\mathrm{MgSO}_{4}, 0.5$. Before sterilization, the $\mathrm{pH}$ of the medium was adjusted to 7.0, and then flasks were sterilized by autoclaving at $121^{\circ} \mathrm{C}$ and $1 \mathrm{~atm}$. After cooling, flasks were inoculated with a loop of the actinomycete isolate previously maintained on starch nitrate agar slants. Inoculated flasks were incubated on a rotary shaker at $32^{\circ} \mathrm{C}$ and 
$200 \mathrm{rpm}$ for $48 \mathrm{~h}$, and the growing vegetative cells were used as standard inoculum.

2.4. Production Medium. The initial production medium was prepared according to Niwas et al. [25] in Erlenmeyer flasks containing $50 \mathrm{~mL}$ of medium. The medium composed of the following $(\mathrm{g} / \mathrm{L})$ : glucose, 4; yeast extract, 4; malt extract, 10; $\mathrm{CaCO}_{3}, 2$; and cholesterol, 2. Cholesterol was used throughout the work as an inducer for CO enzyme production. Upon medium preparation, cholesterol was dissolved in $1 \%$ Triton $\mathrm{X}-100$. The $\mathrm{pH}$ was adjusted at 7.0, and the flasks were autoclaved as previously described. Flasks were inoculated with $2 \mathrm{~mL}$ of vegetative cells from the inoculum prepared earlier. Inoculated flasks were incubated on a rotary shaker incubator at $200 \mathrm{rpm}$ and $32^{\circ} \mathrm{C}$. After the specified incubation time for each set of experimental trials, the mycelial growth was collected by centrifugation at $5000 \mathrm{rpm}$ for $10 \mathrm{~min}$. The cell-free supernatant was used for the determination of the $\mathrm{CO}$ enzyme.

2.5. Identification of Most Significant Nutrients by PlackettBurman Design. Plackett-Burman design (PBD) was used to identify significant nutrients affecting CO production [26]. The four main variables investigated, i.e., glucose, yeast extract, malt extract, and $\mathrm{CaCO}_{3}$, were represented by $X 1$, $X 2, X 3$, and $X 4$, respectively. These variables were selected based on previous literature [25]. The variables were evaluated in two levels, high $(+1)$ and low $(-1)$ levels. Different experimental runs (12 runs) were performed according to the model. PBD experimental design is based on the firstorder polynomial equation:

$$
Y=\beta_{0}+\sum \beta_{i} X_{i}
$$

where $Y$ is the activity of $\mathrm{CO}, \beta_{0}$ is the coefficient of the model, $\beta_{i}$ is the linear coefficient, and $X_{i}$ is the levels of each of the independent factors. From the regression analysis, variables that were significant at or above $95 \%$ level and at probability value of $p<0.05$ were considered to have a great impact on $\mathrm{CO}$ production and were further statistically optimized by BBD.

2.6. Box-Behnken Design Experiments. Following PBD medium optimization, the most significant factors affecting $\mathrm{CO}$ production were evaluated using BBD. Experiments involving four variables resulted in a combination of $27 \mathrm{ex}-$ periments, 4 continuous factors, and 3 replicates at the center point. The variables tested were $X 1$ (glucose), $X 2$ (yeast extract), $X 3$ (malt extract), and $X 4\left(\mathrm{CaCO}_{3}\right)$. Each independent variable was coded in 3 levels $(-1,0$, and +1$)$. The response function $(Y)$ representing $\mathrm{CO}$ activity was partitioned into linear, quadratic, and interactive components, which were represented using the second-order polynomial function as

$$
Y=\beta_{0}+\sum \beta_{i} x_{i}+\text { Oiixi }^{2}+\sum \beta_{i j} X_{i} X_{j}, \quad i=1,2,3, \ldots, k 2,
$$

where $\beta_{0}, \beta_{i}, \beta_{i i}$, and $\beta_{i j}$ are the coefficients of intercept, linear, quadratic, and interactive terms, respectively, while $X_{i}$ and $X_{j}$ are the coded values of the four independent variables under study. The accuracy of the fitted model was justified through the analysis of variance (ANOVA) and the coefficient of $R^{2}$. The significance of all terms in the polynomial model was judged statistically by computing the $F$-value at a probability ( $p$ value) of 0.05 . Minitab software 17.0 was used for the regression of the experimental data and to generate the $2 \mathrm{D}$ contour plots, which were generated by keeping twovariable constants at 0 levels and varying the other variables within the experimental range. The whole set of experiments was performed in triplicate and the mean response was used for analysis.

2.7. Enzyme Assay. The activity of the extracellular CO enzyme was determined according to the method described by Inouye et al. [27]. Briefly, $0.1 \mathrm{~mL}$ of culture supernatant was added to $0.4 \mathrm{~mL}$ of $125 \mathrm{mM}$ Tris- $\mathrm{HCl}$ buffer ( $\mathrm{pH} 7.5$ ). The mixture was incubated in a water bath at $37^{\circ} \mathrm{C}$. After 3 minutes, $25 \mu \mathrm{L}$ of $12 \mathrm{mM}$ of cholesterol in isopropanol solution was added to the mixture, and incubation proceeded for a further 30 minutes. Afterwards, $2.5 \mathrm{~mL}$ of absolute ethanol was added to the reaction medium, and then the amount of formed 4-cholesten-3-one was determined spectrophotometrically by measuring the absorbance at $240 \mathrm{~nm}$. Reaction blanks were prepared by cholesterol solution with isopropanol. One unit of cholesterol oxidase activity (U) was defined as the amount resulting in the formation of $1 \mu \mathrm{mol}$ of 4 -cholesten-3-one in 30 minutes at $37^{\circ} \mathrm{C}$. The concentration of 4 -colesten-3-one was calculated from a standard curve previously prepared with serial dilutions $(10-100 \mu \mathrm{g})$ of 4 -cholesten-3-one dissolved in isopropanol.

\section{Results and Discussion}

3.1. Isolation, Identification, and Molecular Characterization of Cholesterol Oxidase Producing Strain. The first task of the work was to isolate an actinomycete strain capable of producing $\mathrm{CO}$ enzyme. Among different isolated colonies, the most active colony in terms of the highest CO activity was isolated and was further identified on a molecular basis. The isolated actinomycete formed extensive branching in both substrate mycelia and aerial hyphae, which differentiated into long straight chains (Rectiflexibiles type) carrying smooth-surfaced spores (Figure 1). The colony colour varies from white to grey on starch nitrate agar medium. BLAST software was used to compare the partial nucleotide sequence of the $16 \mathrm{~S}$ rRNA gene of the CO-producing isolate with nucleotide databases found in NCBI webserver. Results showed higher similarity ranges (99.31-99.41\%) with different Streptomyces strains, with an E-value of 0.0. From the phylogenetic tree (Figure 2), constructed based on molecular taxonomy and phylogeny, the isolated strain under study was closely related to many Streptomyces sp. [28]. Among nucleotides sequences of the $16 \mathrm{~S}$ rRNA gene that were aligned, the similarity of the potent isolate was $99.4 \%$ to 
TABLE 1: PBD with enzyme activity response as affected by the four independent variables.

\begin{tabular}{lccccc}
\hline Run & $\begin{array}{c}X 1 \\
\text { Glucose }(\mathrm{g} / \mathrm{L})\end{array}$ & $\begin{array}{c}X 2 \\
\text { Yeast extract }(\mathrm{g} / \mathrm{L})\end{array}$ & $\begin{array}{c}X 3 \\
\text { Malt extract }(\mathrm{g} / \mathrm{L})\end{array}$ & $\begin{array}{c}X 4 \\
\mathrm{CaCO}_{3}(\mathrm{~g} / \mathrm{L})\end{array}$ & Response CO activity $(\mathrm{U} / \mathrm{mL})$ \\
\hline 1 & $6.0(+1)$ & $2.0(-1)$ & $12.0(+1)$ & $0.5(-1)$ & 21.732 \\
2 & $6.0(+1)$ & $6.0(+1)$ & $8.00(-1)$ & $4.0(+1)$ & 17.713 \\
3 & $2.0(-1)$ & $6.0(+1)$ & $12.0(+1)$ & $0.5(-1)$ & 10.081 \\
4 & $6.0(+1)$ & $2.0(-1)$ & $12.0(+1)$ & $4.0(+1)$ & 21.940 \\
5 & $6.0(+1)$ & $6.0(+1)$ & $8.00(-1)$ & $4.0(+1)$ & 17.713 \\
6 & $6.0(+1)$ & $6.0(+1)$ & $12.0(+1)$ & $0.5(-1)$ & 18.000 \\
7 & $2.0(-1)$ & $6.0(+1)$ & $12.0(+1)$ & $4.0(+1)$ & 1.597 \\
8 & $2.0(-1)$ & $2.0(-1)$ & $12.0(+1)$ & $4.0(+1)$ & 16.761 \\
9 & $2.0(-1)$ & $2.0(-1)$ & $8.00(-1)$ & $4.0(+1)$ & 21.245 \\
10 & $6.0(+1)$ & $2.0(-1)$ & $8.00(-1)$ & $0.5(-1)$ & 8.6070 \\
11 & $2.0(-1)$ & $6.0(+1)$ & $8.00(-1)$ & $0.5(-1)$ & 12.870 \\
12 & $2.0(-1)$ & $2.0(-1)$ & $8.00(-1)$ & $0.5(-1)$ & \\
\hline
\end{tabular}

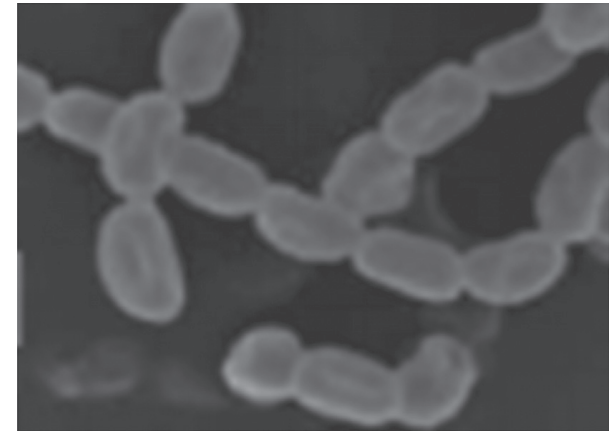

FIGURE 1: Spore chain morphology of Streptomyces rochei NAM-19 showing smooth surfaces in Rectiflexibiles (RF) chains by SEM $(15 \mathrm{Kv} \times 10,000)$.

Streptomyces rochei NRRL 1559. Hence, the strain was identified as a novel Streptomyces rochei NAM-19, and the $16 \mathrm{~S}$ rRNA sequence was deposited in the GenBank database under accession number MN630193.

3.2. Evaluation of the Most Significant Factors Affecting CO Activity. The optimization of key nutrients in fermentation medium for CO production by $S$. rochei NAM-19 using PBD was carried out in submerged cultures. Table 1 presents different investigated variables with their high and low levels, as well as their obtained response in the activity of CO enzyme. It can be seen that the obtained $\mathrm{CO}$ activities ranged from 8.06 to $21.94 \mathrm{U} / \mathrm{mL}$. Furthermore, the maximal enzyme production response was obtained in runs 1,4 , and 10 (21.46-21.94 U/mL). The variation reflected the significance of glucose, yeast extract, malt extract, and $\mathrm{CaCO}_{3}$ on $\mathrm{CO}$ production by the isolated strain. Maximal $\mathrm{CO}$ production was obtained in the $4^{\text {th }}$ run, which may be attributed to the presence of high levels of glucose, malt extract, and $\mathrm{CaCO}_{3}$ and low level of yeast extract. On the other hand, decreased $\mathrm{CO}$ production was correlated with inversed patterns of the four investigated medium components. It is generally known that combination of medium ingredients has a profound influence on the metabolic pathways of the producing organisms that regulate the production of different metabolites [29]. Various compounds such as cholesterol, yeast extract [30], potato starch, peptone, and malt extract [31] have been recorded to be substrates for an enhanced CO production.

The adequacy of the model was tested and parameters with statistically significant effects were identified using Fisher's (F) test for the analysis of variance (ANOVA). The analysis of variance for the selected factorial model showed that the model was significant with a Model F-value of 101.06 and $p$ value of 0.000 , which means that the above model is reliable enough to describe the quantitative relation between $\mathrm{CO}$ production and the four important medium components (Table 2).

The regression equation obtained from $\mathrm{PBD}$ was used to predict the factors affecting the $\mathrm{CO}$ activity response. The equation was expressed by $R^{2}$ coefficient, which was about $98.30 \%$ successful in predicting the effects of the variables on CO production by $S$. rochei NAM-19. Therefore, all investigated factors were significant $(p \leq 0.05)$ and the model equation for enzyme production can be represented as

$$
\begin{aligned}
\operatorname{CO} \operatorname{activity}(\mathrm{U} / \mathrm{mL})= & 9.70+1.808 X 1-1.096 X 2 \\
& +0.271 X 3+0.391 X 4,
\end{aligned}
$$

where positive signs before the coefficient values, in case of glucose, malt extract, and $\mathrm{CaCO}_{3}$, indicate the significance of these factors at their high levels towards $\mathrm{CO}$ production. This indicated that CO production was enhanced by adding a higher concentration of these ingredients, whereas the negatively signed yeast extract has an inverse relationship with CO production (Table 3 ).

Figure 3 represents the main effects of different factors on $\mathrm{CO}$ production obtained from PBD. The main effect enables the estimation of the influence of each factor on enzyme production. Both large positive or negative effects indicate that a variable has a large impact on production. From the main effect results, it can be noticed that glucose, malt extract, and $\mathrm{CaCO}_{3}$ have a positive influence on enzyme production, whereas yeast extract negatively affects $\mathrm{CO}$ production. Variables with positive impacts on $\mathrm{CO}$ production have been used at their high levels, while variables with a negative effect were kept at their low level for further optimization. 


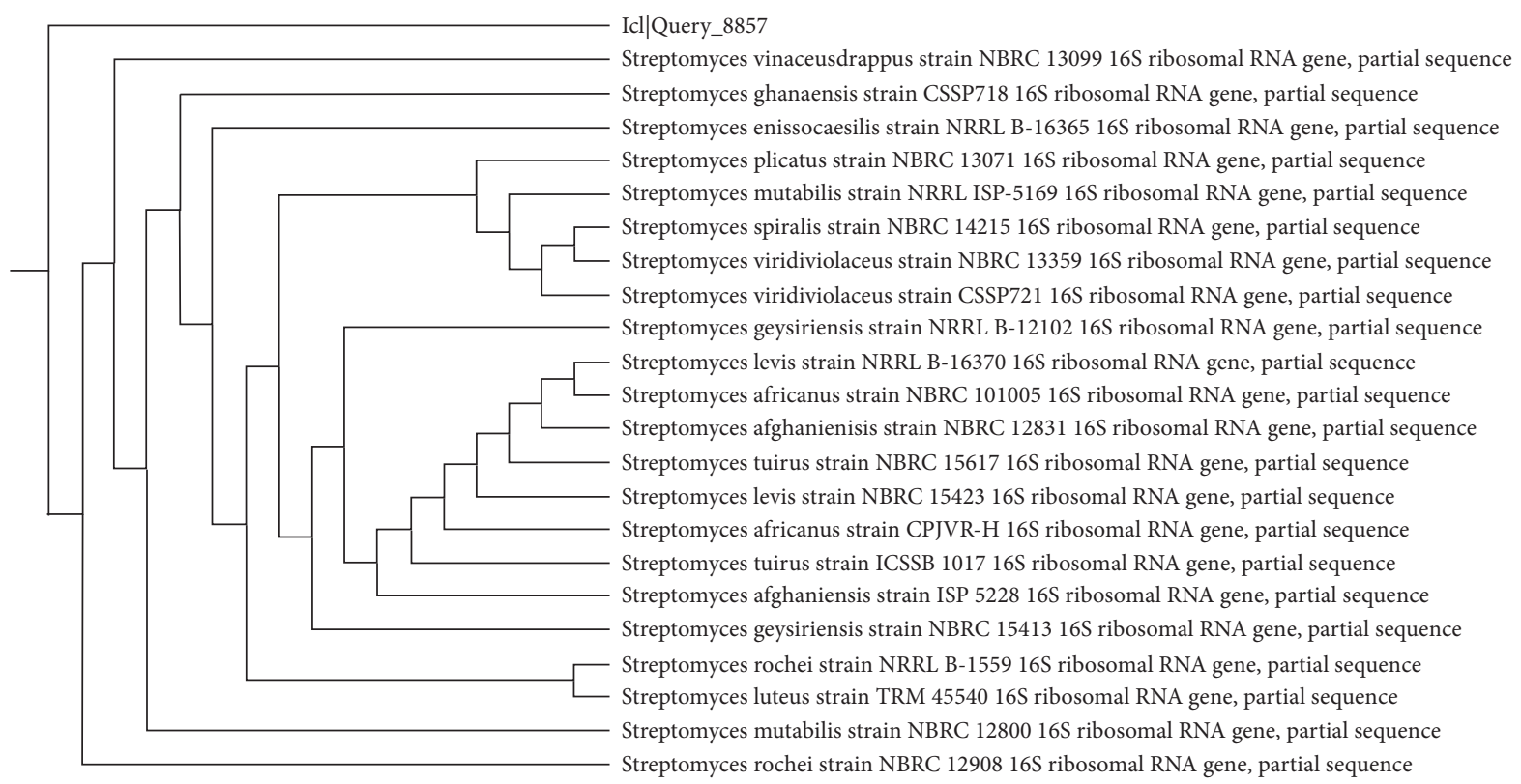

FIGURE 2: Neighbor-joining phylogenetic representation of the strains and their closest relatives based on 16S rRNA gene sequences of $S$. rochei NAM-19.

TABLE 2: Analysis of variance of $\mathrm{CO}$ activity versus glucose, yeast extract, malt extract, and $\mathrm{CaCO}_{3}$.

\begin{tabular}{|c|c|c|c|c|c|}
\hline Source & $\mathrm{DF}$ & Adj. SS & Adj. MS & $F$-value & $p$ value \\
\hline Model & 4 & 223.723 & 55.931 & 101.06 & 0.000 \\
\hline Linear & 4 & 223.723 & 55.931 & 101.06 & 0.000 \\
\hline Glucose $(X 1)$ & 1 & 156.942 & 156.942 & 283.56 & 0.000 \\
\hline Yeast extract $(X 2)$ & 1 & 57.628 & 57.628 & 104.12 & 0.000 \\
\hline Malt extract $(X 3)$ & 1 & 3.524 & 3.524 & 6.37 & 0.040 \\
\hline $\mathrm{CaCO}_{3}(X 4)$ & 1 & 5.629 & 5.629 & 10.17 & 0.015 \\
\hline Residual error & 7 & 3.874 & 0.553 & & \\
\hline Lack-of-fit & 6 & 3.874 & 0.646 & & \\
\hline Pure error & 1 & 0.000 & 0.000 & & \\
\hline Corrected total & 11 & 227.597 & & & \\
\hline Model summary & S & R-seq. & R-seq. (Adjusted) & R-seq. (Predicted) & \\
\hline & 0.743952 & $98.30 \%$ & $97.33 \%$ & $95.00 \%$ & \\
\hline
\end{tabular}

TABLE 3: Regression analysis of PBD with model coefficients and significance of the regression coefficient for $\mathrm{CO}$ activity.

\begin{tabular}{lccccc}
\hline Term & Effect & Coefficient & SE coefficient & $T$-value & $p$ value \\
\hline Constant & & 9.700 & 1.280 & 7.570 & 0.000 \\
$X 1$ & 3.616 & 1.808 & 0.107 & 16.84 & 0.000 \\
$X 2$ & -2.191 & -1.096 & 0.107 & -10.20 & 0.000 \\
$X 3$ & 0.542 & 0.271 & 0.107 & 2.500 & 0.040 \\
$X 4$ & 0.783 & 0.391 & 0.123 & 3.19 & 0.015 \\
\hline
\end{tabular}

In order to confirm model adequacy, residual analysis plots (Figure 4) were generated. The normality of the data can be checked by plotting the normal probability plot (NPP) of the residuals. The normal probability plot is a graphical representation for assessing whether or not a data set is approximately normally distributed [32]. The residual is the difference between the observed and the predicted

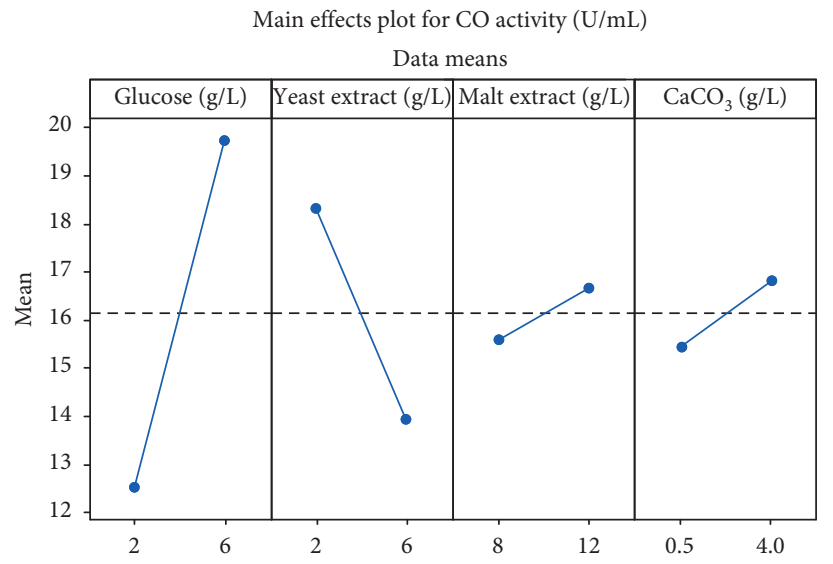

FIgURE 3: Effect of glucose, yeast extract, malt extract, and $\mathrm{CaCO}_{3}$ concentrations on the mean enzyme activity represented by the main effect plot. 


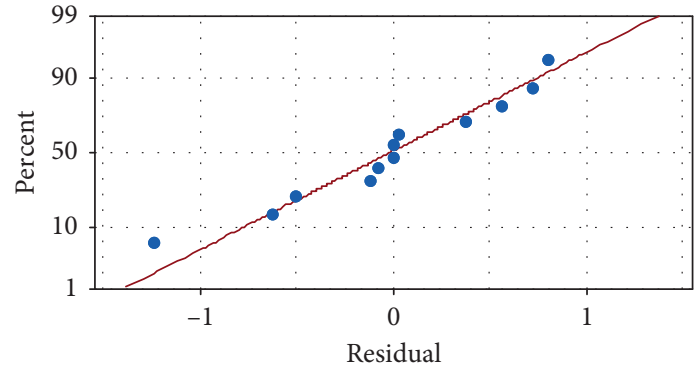

(a)

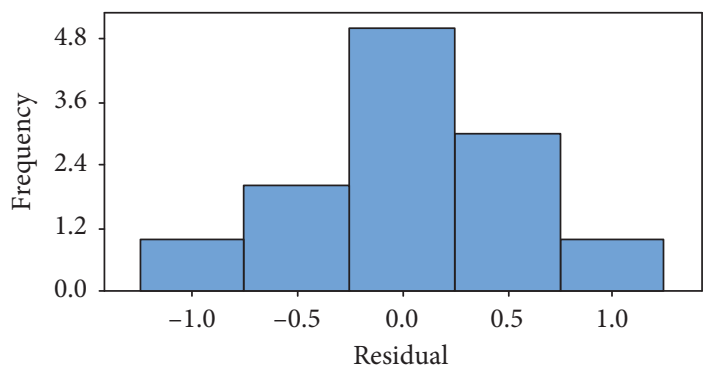

(c)

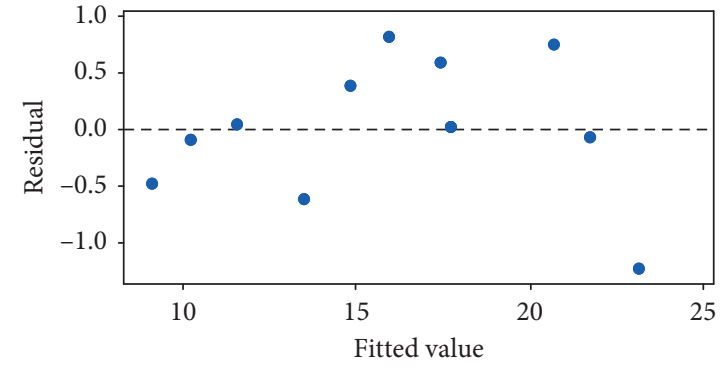

(b)

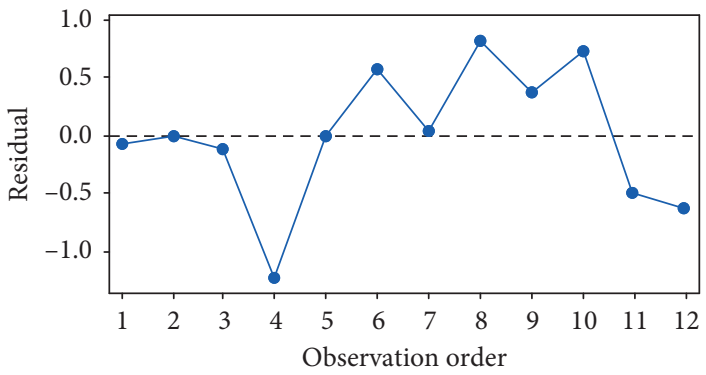

(d)

Figure 4: Residual plots for CO activity. (a) Normal Probability Plot. (b) Versus Fits. (c) Histogram. (d) Versus Order.

value (or the fitted value) from the regression. It could be seen that the experimental data points were approximately linear, suggesting normal distribution, which indicated that the model can be used to optimize the production of CO. Histograms of the residuals showed an almost symmetrical histogram (bell-shaped, i.e., the errors are normally distributed with mean zero). Regarding the plots of residuals versus the fitted values (predicted response), the residuals are scattered randomly about zero; i.e., errors have constant variance and all other points were found to fall in the range of +1 to -1 .

Pareto chart (Figure 5) represents the estimated effects of variables on enzyme activity response in decreasing the order of magnitude. The length of each bar is proportional to the standardized effect. The vertical line can be used to judge which effects are statistically significant. Bars extending beyond this line correspond to statistically significant effects at a confidence level of 95\% [33]. It can be seen that all independent variables had a significant effect towards enzyme activity response. Additionally, significant factors were further confirmed by the normal plotting of standardized effects (Figure 6). It can be seen that the three significant variables have higher percentages and are lying together on the right-hand side of the standardized effect line. Accordingly, the four variables were further considered for final optimization using BBD.

3.3. Response Surface Experimental Design Using BBD. The optimization of medium key components was further evaluated using RSM by BBD. This was performed to determine the true optimal concentrations of the key variables affecting $\mathrm{CO}$ enzyme production and to analyse the interactive effects of their concentrations. For these four

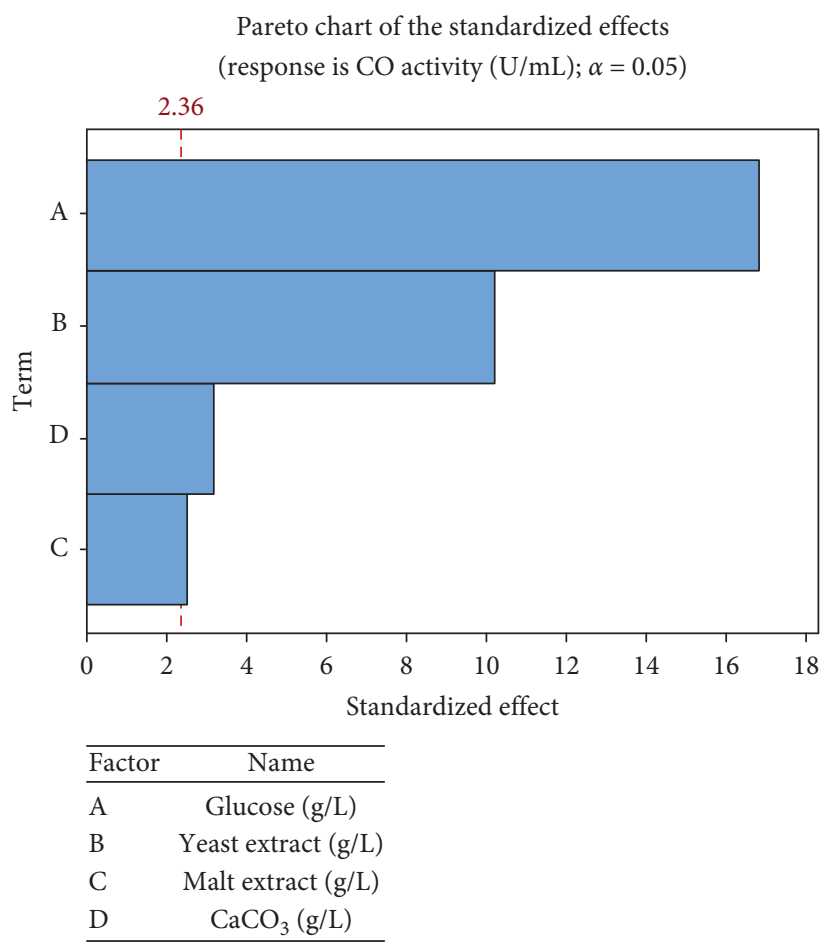

FIGURE 5: Pareto chart for the effect of medium nutrient components according to $\mathrm{PBD}$.

variables, a design matrix of 27 runs with three levels $(-1$, low; 0 , middle; +1 , high) of each variable was constructed. Table 4 presents the combinations of these 27 runs with their obtained enzyme activity responses. From these results, it can be seen that maximal enzyme activity response of $57.73 \mathrm{U} / \mathrm{mL}$ was obtained in run 18 , which contained higher 


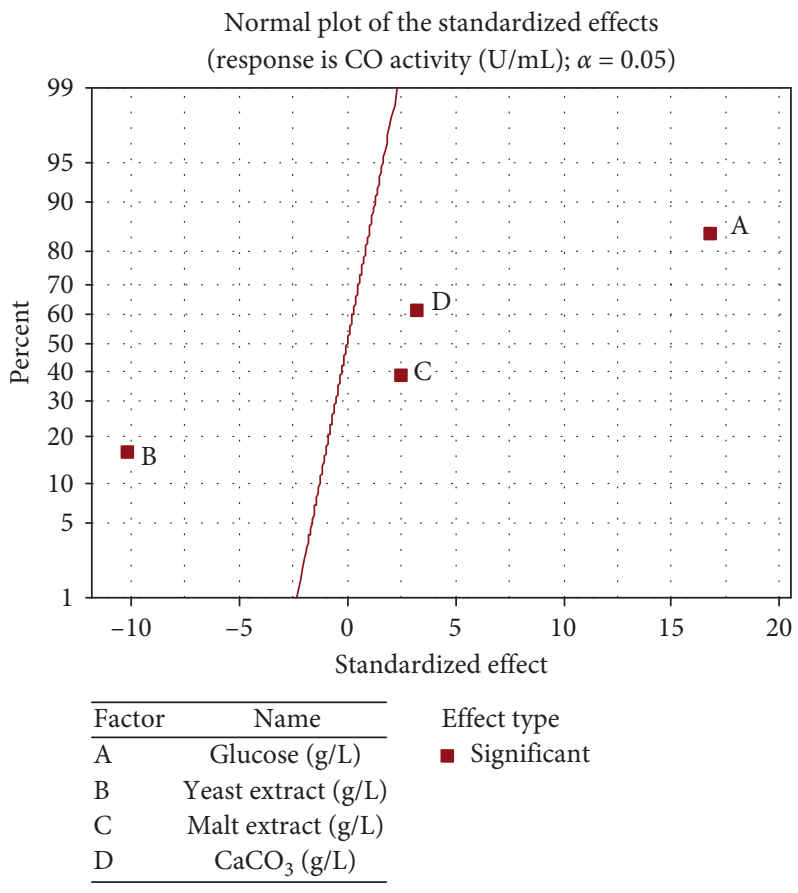

Figure 6: Normal plot of the standardized effects of components on CO enzyme activity.

TABLE 4: BBD representing the predicted CO production by S. rochei NAM-19. Levels of coded variables are in parenthesis.

\begin{tabular}{|c|c|c|c|c|c|}
\hline Run & $\begin{array}{c}X 1 \\
\text { Glucose }(\mathrm{g} / \mathrm{L}) \\
\end{array}$ & $\begin{array}{c}X 2 \\
\text { Yeast extract }(\mathrm{g} / \mathrm{L}) \\
\end{array}$ & $\begin{array}{c}X 3 \\
\text { Malt extract }(\mathrm{g} / \mathrm{L}) \\
\end{array}$ & $\begin{array}{c}X 4 \\
\mathrm{CaCO}_{3}(\mathrm{~g} / \mathrm{L}) \\
\end{array}$ & Response CO activity $(\mathrm{U} / \mathrm{mL})$ \\
\hline$\overline{1}$ & $4(-1)$ & $0(-1)$ & $12(0)$ & $4(0)$ & 26.300 \\
\hline 2 & $8(+1)$ & $0(-1)$ & $12(0)$ & $4(0)$ & 43.600 \\
\hline 3 & $4(-1)$ & $4(+1)$ & $12(0)$ & $4(0)$ & 34.040 \\
\hline 4 & $8(+1)$ & $4(+1)$ & $12(0)$ & $4(0)$ & 54.000 \\
\hline 5 & $6(0)$ & $2(0)$ & $10(-1)$ & $2(-1)$ & 42.400 \\
\hline 6 & $6(0)$ & $2(0)$ & $14(+1)$ & $2(-1)$ & 36.000 \\
\hline 7 & $6(0)$ & $2(0)$ & $10(-1)$ & $6(+1)$ & 43.440 \\
\hline 8 & $6(0)$ & $2(0)$ & $14(+1)$ & $6(+1)$ & 35.840 \\
\hline 9 & $4(-1)$ & $2(0)$ & $12(0)$ & $2(-1)$ & 33.640 \\
\hline 10 & $8(+1)$ & $2(0)$ & $12(0)$ & $2(-1)$ & 49.240 \\
\hline 11 & $4(-1)$ & $2(0)$ & $12(0)$ & $6(+1)$ & 31.100 \\
\hline 12 & $8(+1)$ & $2(0)$ & $12(0)$ & $6(+1)$ & 52.700 \\
\hline 13 & $6(0)$ & $0(-1)$ & $10(-1)$ & $4(0)$ & 36.300 \\
\hline 14 & $6(0)$ & $4(+1)$ & $10(-1)$ & $4(0)$ & 45.200 \\
\hline 15 & $6(0)$ & $0(-1)$ & $14(+1)$ & $4(0)$ & 29.140 \\
\hline 16 & $6(0)$ & $4(+1)$ & $14(+1)$ & $4(0)$ & 38.340 \\
\hline 17 & $4(-1)$ & $2(0)$ & $10(-1)$ & $4(0)$ & 33.880 \\
\hline 18 & $8(+1)$ & $2(0)$ & $10(-1)$ & $4(0)$ & 57.730 \\
\hline 19 & $4(-1)$ & $2(0)$ & $14(+1)$ & $4(0)$ & 32.120 \\
\hline 20 & $8(+1)$ & $2(0)$ & $14(+1)$ & $4(0)$ & 45.500 \\
\hline 21 & $6(0)$ & $0(-1)$ & $12(0)$ & $2(-1)$ & 34.200 \\
\hline 22 & $6(0)$ & $4(+1)$ & $12(0)$ & $2(-1)$ & 38.600 \\
\hline 23 & $6(0)$ & $0(-1)$ & $12(0)$ & $6(+1)$ & 29.976 \\
\hline 24 & $6(0)$ & $4(+1)$ & $12(0)$ & $6(+1)$ & 43.700 \\
\hline 25 & $6(0)$ & $2(0)$ & $12(0)$ & $4(0)$ & 41.500 \\
\hline 26 & $6(0)$ & $2(0)$ & $12(0)$ & $4(0)$ & 40.300 \\
\hline 27 & $6(0)$ & $2(0)$ & $12(0)$ & $4(0)$ & 39.500 \\
\hline
\end{tabular}

level of glucose $(8 \mathrm{~g} / \mathrm{L})$, middle levels of yeast extracts and calcium carbonate ( 2 and $4 \mathrm{~g} / \mathrm{L}$, respectively), and lower level of malt extract $(10 \mathrm{~g} / \mathrm{L})$.
The statistical significance of the model is determined by $F$-value obtained from ANOVA analysis (Table 5). A large Fisher's value $(F$-value $=7500.08)$ indicates that most of the 
TABLe 5: Analysis of variance of BBD for CO production model using S. rochei NAM-19.

\begin{tabular}{|c|c|c|c|c|c|}
\hline Source & DF & Adj. SS & Adj. MS & $F$-value & $p$ value \\
\hline Model & 14 & 1586.49 & 113.21 & 7500.08 & 0.000 \\
\hline Linear & 4 & 8.72 & 2.181 & 144.34 & 0.000 \\
\hline$X 1$ & 1 & 6.36 & 6.360 & 420.90 & 0.000 \\
\hline$X 2$ & 1 & 0.88 & 0.878 & 58.19 & 0.000 \\
\hline$X 3$ & 1 & 4.31 & 4.313 & 285.44 & 0.000 \\
\hline$X 4$ & 1 & 0.00 & 0.000 & 0.03 & 0.870 \\
\hline Square & 4 & 110.93 & 27.733 & 1835.51 & 0.000 \\
\hline$X 1^{2}$ & 1 & 16.93 & 16.933 & 1120.70 & 0.000 \\
\hline$X 2^{2}$ & 1 & 57.20 & 57.200 & 3785.77 & 0.000 \\
\hline$X 3^{2}$ & 1 & 1.62 & 1.617 & 107.04 & 0.000 \\
\hline$X 4^{2}$ & 1 & 7.46 & 7.457 & 493.51 & 0.000 \\
\hline 2-Way interaction & 6 & 61.34 & 10.224 & 676.67 & 0.000 \\
\hline$X 1 * X 2$ & 1 & 2.82 & 2.822 & 186.80 & 0.000 \\
\hline$X 1 * X 3$ & 1 & 27.41 & 27.405 & 1813.80 & 0.000 \\
\hline$X 1 * X 4$ & 1 & 9.00 & 9.000 & 595.66 & 0.000 \\
\hline$X 2 * X 3$ & 1 & 0.02 & 0.022 & 1.49 & 0.246 \\
\hline$X 2 * X 4$ & 1 & 21.73 & 21.734 & 1438.47 & 0.000 \\
\hline$X 3 * X 4$ & 1 & 0.36 & 0.360 & 23.83 & 0.000 \\
\hline Residual error & 12 & 0.18 & 0.015 & & \\
\hline Pure error & 2 & 0.00 & 0.000 & & \\
\hline Corrected total & 26 & 1586.68 & \multirow{2}{*}{\multicolumn{2}{|c|}{$\begin{array}{c}\text { R-seq. (Adjusted) } \\
99.98 \%\end{array}$}} & \\
\hline Model summary & $\begin{array}{c}\mathrm{S} \\
0.122920\end{array}$ & $\begin{array}{l}\text { R-seq. } \\
99.99 \%\end{array}$ & & & $\begin{array}{c}\text { R-seq. (predicted) } \\
99.93 \%\end{array}$ \\
\hline
\end{tabular}

variation can be explained by a regression equation, whereas a low $p$ value $(p<0.005)$ indicates the statistical significance of the model. Regression coefficient $\left(R^{2}\right)$ came out to be $99.99 \%$, the predicted $R$-squared (99.93\%), and the adjusted $R$-squared (99.98\%), which were in a reasonable argument with each other. Looking at the obtained $p$ values for the model, results showed that the linear terms $X 1, X 2$, and $X 3$, all quadratic terms $X 1^{2}, X 2^{2}, X 3^{2}$, and $X 4^{2}$, and cross terms $X 1 * X 2, X 1 * X 3, X 1 * X 4, X 2 * X 4$, and $X 3 * X 4$ were highly significant.

Furthermore, Table 6 presents the regression analysis of $\mathrm{BBD}$. It can be seen that the interaction between two variables can have either a synergetic effect, which is a positive coefficient increasing CO production, or an antagonistic effect, which is a negative coefficient indicating a decrease in $\mathrm{CO}$ production. Moreover, the analysis showed a significant negative quadratic effect of yeast extract and $\mathrm{CaCO}_{3}$, indicating that $\mathrm{CO}$ production increases with the increase of these parameters, then, reaches a maximum, and finally decreases at even higher concentrations of both variables (Table 6).
Generally, $\mathrm{CaCO}_{3}$ is used as a buffering agent in the production medium of most of the actinomycete strains, due to its high buffering capacity. However, the statistical optimization results showed that it is not significant. This could be attributed to the effect of $\mathrm{Ca}^{+2}$ ions on the morphology of the growing cells and consequently on $\mathrm{CO}$ production. It has been reported that addition of $\mathrm{Ca}^{+2}$ ions tends to decrease the probability of formation of pellets and enhances the formation of dispersed mycelia [34]. Moreover, the formed fewer pellets were of reduced size $[35,36]$. This effect on the morphology of growing cells was reflected in the reduced production of peroxidase, protease, and oxidases by Streptomyces sp. However, we preferred not to remove $\mathrm{CaCO}_{3}$ from the production medium in order to benefit from its buffering capacity, which in turn provides the growing cells with their optimal required conditions.

Results obtained from BBD experimental runs were used to estimate the coefficients of the quadratic polynomial equation. The second-order polynomial equation characterizing relationships between $\mathrm{CO}$ production and different variables can be expressed as

$$
\begin{aligned}
\operatorname{CO} \operatorname{activity}(\mathrm{U} / \mathrm{mL})= & -16.44+5.212 X 1+1.695 X 2+5.742 X 3-0.039 X 4+0.4455 X 12-0.8187 X 22-0.1377 X 32-0.2956 X 42 \\
& +0.2100 X 1 * X 2-0.6544 X 1 * X 3+0.3750 X 1 * X 4+0.0187 X 2 * X 3+0.5828 X 2 * X 4-0.0750 X 4 * X 4
\end{aligned}
$$

Figures 7(a)-7(f) represent 2D contour plots of different relationships between independent and dependent variables of the model. It is clearly noticed that different contours indicate different interactions between the investigated 
TABLE 6: Regression analysis of BBD with model coefficients for total CO production by S. rochei NAM-19.

\begin{tabular}{|c|c|c|c|c|c|}
\hline Term & Effect & Coefficient & SE coefficient & $T$-value & $p$ value \\
\hline Constant & & -16.44 & 2.63 & -6.26 & 0.000 \\
\hline$X 1$ & 10.423 & 5.212 & 0.254 & 20.52 & 0.000 \\
\hline$X 2$ & 3.390 & 1.695 & 0.222 & 7.62 & 0.000 \\
\hline$X 3$ & 11.485 & 5.742 & 0.340 & 16.89 & 0.000 \\
\hline$X 4$ & -0.078 & -0.039 & 0.235 & -0.17 & 0.870 \\
\hline$X 1^{2}$ & 0.8909 & 0.4455 & 0.0133 & 33.48 & 0.000 \\
\hline$X 2^{2}$ & -1.6375 & -0.8187 & 0.0133 & -61.53 & 0.000 \\
\hline$X 3^{2}$ & -0.2753 & -0.1377 & 0.0133 & -10.35 & 0.000 \\
\hline$X 4^{2}$ & -0.5912 & -0.2956 & 0.0133 & -22.22 & 0.000 \\
\hline$X 1 X 2$ & 0.4200 & 0.2100 & 0.0514 & 13.67 & 0.000 \\
\hline$X 1 X 3$ & -1.3087 & -0.6544 & 0.0514 & -42.59 & 0.000 \\
\hline$X 1 X 4$ & 0.7500 & 0.3750 & 0.0514 & 24.41 & 0.000 \\
\hline$X 2 X 3$ & 0.0375 & 0.0187 & 0.0514 & 1.22 & 0.246 \\
\hline$X 2 X 4$ & 1.1655 & 0.5828 & 0.0514 & 37.93 & 0.000 \\
\hline$X 3 X 4$ & -0.1500 & -0.0750 & 0.0514 & -4.88 & 0.000 \\
\hline
\end{tabular}

variables. Generally, a circular contour plot means negligible interactions between the corresponding variables, while elliptical contours suggest the presence of a significant interaction between the corresponding variables. In our case, two variables were depicted in the contour plot, while the other two variables were fixed at zero levels (their preset middle concentration). Figure 7(a) shows the effect of interaction between $\mathrm{CaCO}_{3}$ and glucose concentration on $\mathrm{CO}$ production. Increasing glucose concentration up to $8 \mathrm{~g} / \mathrm{L}$ and $\mathrm{CaCO}_{3}$ from 3 to $6(\mathrm{~g} / \mathrm{L})$ resulted in maximal $\mathrm{CO}$ production above $50 \mathrm{U} / \mathrm{mL}$. However, below these values, there was a gradual decline in $\mathrm{CO}$ production. On the other hand, increasing $\mathrm{CaCO}_{3}$ concentration from 3.5 to $5 \mathrm{~g} / \mathrm{L}$ and fixing malt extract concentration at $10 \mathrm{~g} / \mathrm{L}$ increased $\mathrm{CO}$ production above $43.5 \mathrm{U} / \mathrm{mL}$ (Figure $7(\mathrm{~d})$ ). Furthermore, increasing malt concentration and decreasing $\mathrm{CaCO}_{3}$ concentration from these optimal levels resulted in a gradual decrease in $\mathrm{CO}$ production.

It may be also observed that higher yeast extract concentrations ( 3 to $4 \mathrm{~g} / \mathrm{L}$ ) combined with increasing $\mathrm{CaCO}_{3}$ concentration from 4 to $6 \mathrm{~g} / \mathrm{L}$ resulted in an increase in enzyme production over $42 \mathrm{U} / \mathrm{mL}$. On the other hand, below these optimal concentrations, a decrease in CO production was obtained (Figure 7(e)). Similar enhancement effects of higher levels of glucose and yeast extract showed a strong positive interaction effect on CO production as shown in Figure 7(f). From the contour plots in Figure 7(b) and 7(c) in Figure 6 , it can be concluded that higher levels of glucose and yeast extract while keeping malt extract concentration at lower levels resulted in an improved CO production.

3.4. Interpretation of Process Optimization Curves. RSM optimization is generally used to identify factor settings, which optimize the required response (CO production). In the present study, the goal for maximization of $\mathrm{CO}$ production by $S$. rochei NAM-19 was to obtain an enzyme production level, which is approximate to the statistically targeted concentration of $62.83 \mathrm{U} / \mathrm{mL}$. Results showed that glucose, yeast extract, and $\mathrm{CaCO}_{3}$ concentrations lower than
8,4 , and $6 \mathrm{~g} / \mathrm{L}$, respectively, together with malt extract concentration above $10 \mathrm{~g} / \mathrm{L}$, did not satisfy this requirement. After RSM optimization experiments, the best combination of factor settings for achieving the desired response was found to be as follows (g/L): glucose, 8 ; yeast extract, 4 ; malt extract, 10; and $\mathrm{CaCO}_{3}, 6$. These concentrations produced the predicted response of $62.83 \mathrm{U} / \mathrm{mL}$ with a desirability score of 1 (Figure 8). In general, optimization plots are used to obtain the predicted response with higher desirability score, to lower-cost factor settings with near-optimal properties, and to study the sensitivity of response variables to changes in the factor settings $[20,21]$.

3.5. Validation of the Model. Finally, to check the accuracy of the model, cultivation runs were performed to compare CO production under both initial medium composition and the final statistically optimized medium composition. Obtained results (Figure 9) showed that cultivation of S. rochei NAM19 using the statistically optimized medium composition resulted in maximal production of $65.1 \mathrm{U} / \mathrm{mL}$ from the CO enzyme. This volumetric production level was closely related to the predicted response $(62.8 \mathrm{U} / \mathrm{mL})$. Furthermore, medium optimization increased maximal $\mathrm{CO}$ production by about 2.55 times the maximal CO production obtained using initial unoptimized medium $(25.5 \mathrm{U} / \mathrm{mL})$. Therefore, experimental runs prove that the model can be validated. RSM optimization has been applied to investigate the interrelationship between different medium components affecting the cell growth and production kinetics of many industrially microbial products $[16,20]$. Furthermore, the obtained RSM optimization results are in good consistency with those previously reported for medium optimization by RSM for cholesterol oxidase production $[37,38]$. The authors optimized medium composition by RSM approaches for CO production by S. badius and S. lavendulae NCIM 2499. The authors were able to achieve an increase in volumetric enzyme production by 2.48 and 2 times their initially used production media. Additionally, the newly isolated Streptomyces rochei NAM-19 showed initial CO production 


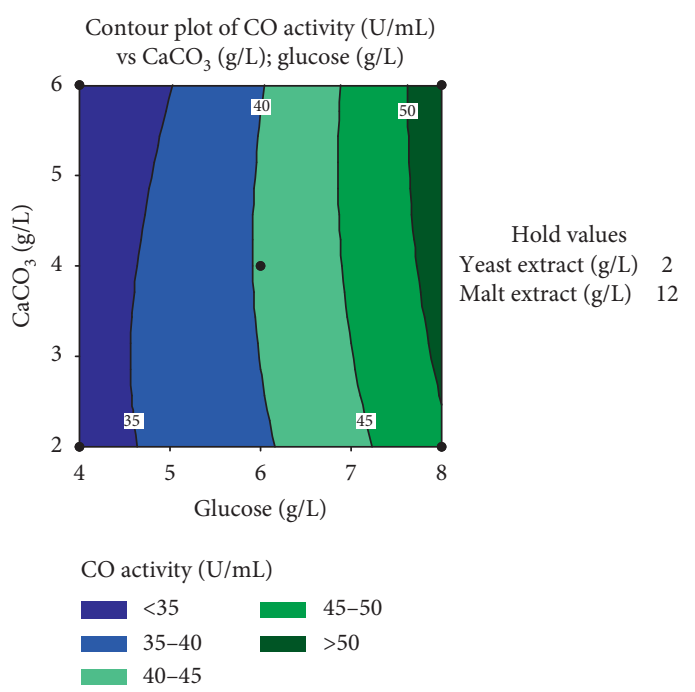

(a)

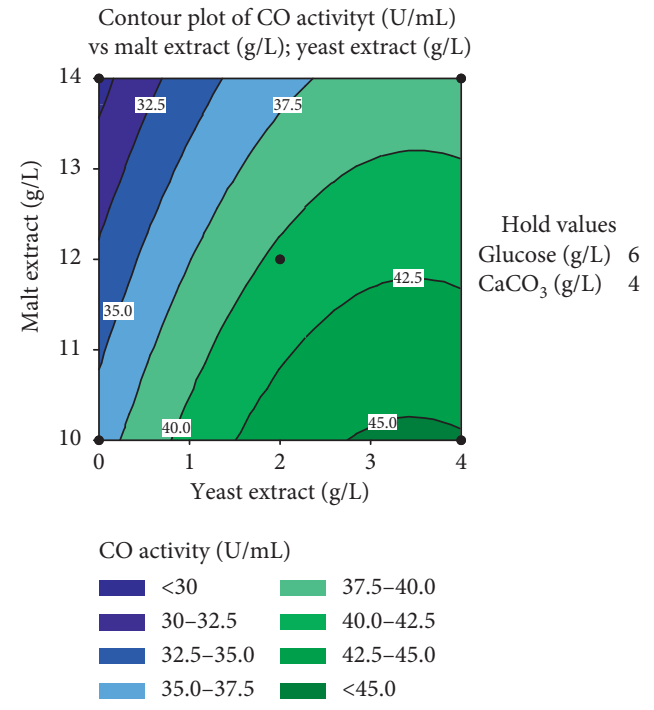

(c)

Contour plot of $\mathrm{CO}$ activityt $(\mathrm{U} / \mathrm{mL})$ vs $\mathrm{CaCO}_{3}(\mathrm{~g} / \mathrm{L})$; yeast extract $(\mathrm{g} / \mathrm{L})$

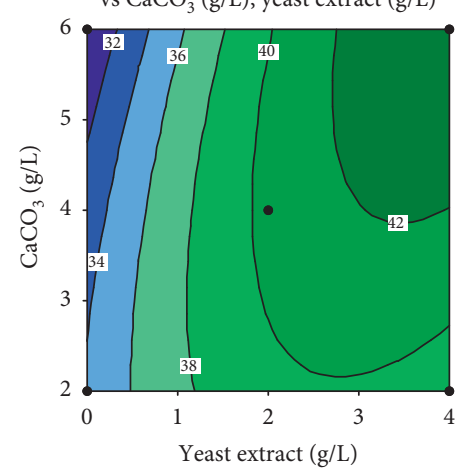

$\mathrm{CO}$ activity $(\mathrm{U} / \mathrm{mL})$

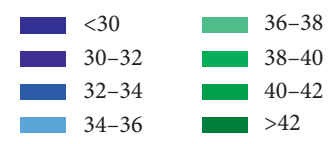

(e)

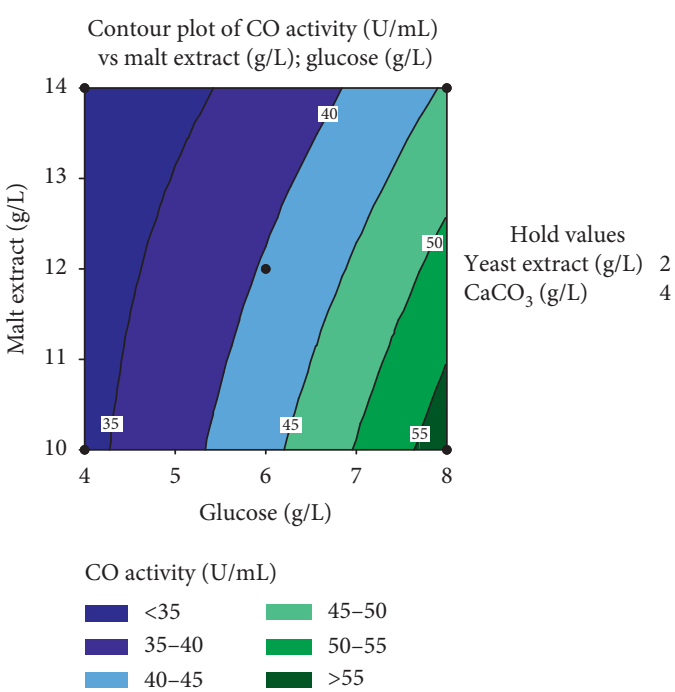

(b)

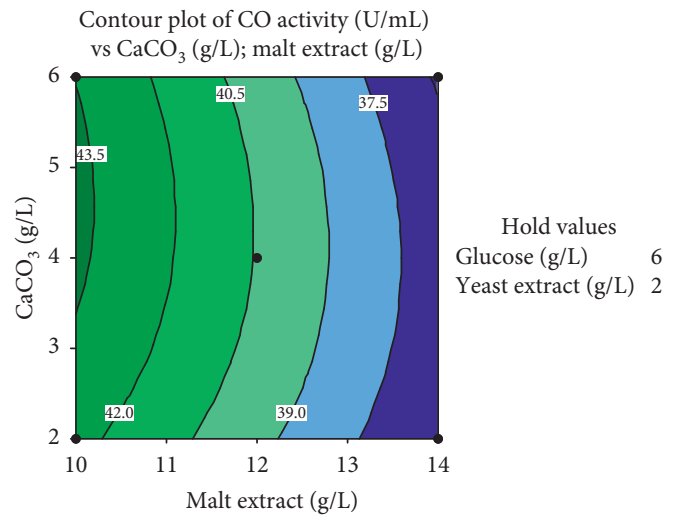

$\mathrm{CO}$ activity $(\mathrm{U} / \mathrm{mL})$

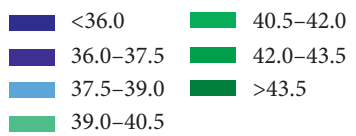

(d)

Contour plot of $\mathrm{CO}$ activity $(\mathrm{U} / \mathrm{mL})$ vs yeast extract $(\mathrm{g} / \mathrm{L})$; glucose $(\mathrm{g} / \mathrm{L})$

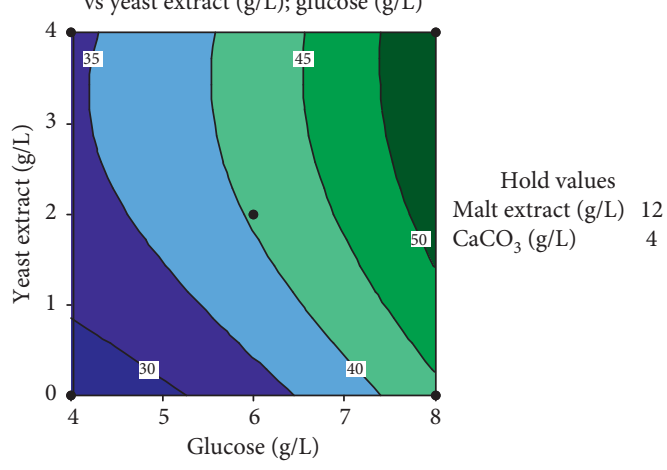

$\mathrm{CO}$ activity $(\mathrm{U} / \mathrm{mL})$

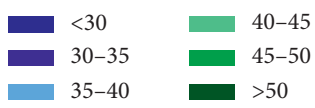

(f)

Figure 7: Contour plots for $\mathrm{CO}$ production as a function of (a) $\mathrm{CaCO}_{3}$ and glucose concentration, (b) glucose and malt extract concentration, (c) yeast extract and malt extract concentration, (d) malt extract and $\mathrm{CaCO}_{3}$ concentration, (e) yeast extract and $\mathrm{CaCO}_{3}$ concentration, and (f) yeast extract and glucose concentration. 


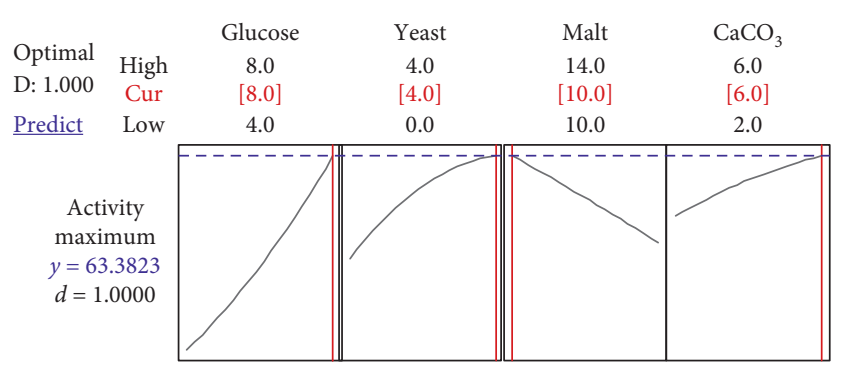

Figure 8: Process optimization curve for maximum enzyme activity.

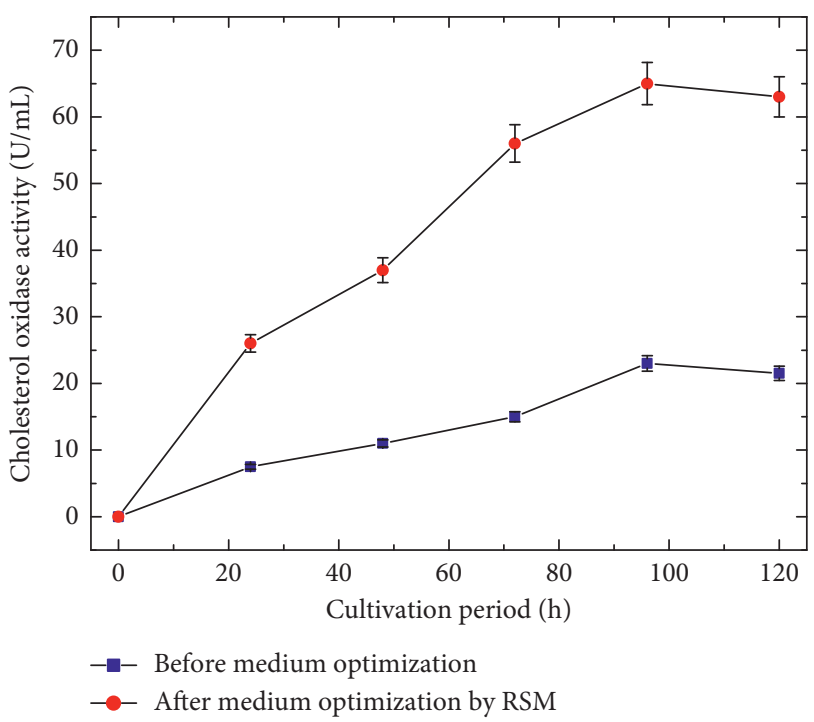

Figure 9: Comparative CO production in the cultivation medium before and after optimization through RSM by S. rochei NAM-19 strain.

levels, which were comparable to those previously published in the literature by Streptomyces sp. [39].

\section{Conclusion}

During the current work, a new strain showing promising potential for cholesterol oxidase production was isolated from soil. The isolated strain was identified with the help of $16 \mathrm{~S}$ rRNA and molecular taxonomy approaches as $S$. rochei NAM-19. The genomic sequence of the isolated strain was deposited in the NCBI database under the accession number MN630193. Furthermore, Plackett-Burman optimization showed that the initial concentration of glucose, yeast extract, malt extract, and $\mathrm{CaCO}_{3}$ significantly affected enzyme production. Additionally, Box-Behnken design revealed that the composition $(\mathrm{g} / \mathrm{L})$ of the key medium variables influencing enzyme production is as follows: glucose, 8; yeast extract, 4; malt extract, 10 ; and $\mathrm{CaCO}_{3}, 6$. The applied optimization model gave a predicted enzyme concentration of $62.83 \mathrm{U} / \mathrm{mL}$ with a desirability score of 1 , which was very close to the experimentally obtained volumetric production level $(65.1 \mathrm{U} / \mathrm{mL})$. The application of
RSM approach increased total enzyme production by about 2.55 times the initially unoptimized medium composition $(25.5 \mathrm{U} / \mathrm{mL})$.

\section{Data Availability}

All data generated in this current work are included in the Results and Discussion sections.

\section{Conflicts of Interest}

The authors declare that the research was conducted in the absence of any commercial or financial relationships that could be construed as potential conflicts of interest.

\section{Acknowledgments}

The authors are grateful to King Saud University, Riyadh, Saudi Arabia, for funding the work through Researchers Supporting Project (Project no. RSP-2019/52).

\section{References}

[1] J. MacLachlan, A. T. L. Wotherspoon, R. O. Ansell, and C. J. W. Brooks, "Cholesterol oxidase: sources, physical properties and analytical applications," The Journal of Steroid Biochemistry and Molecular Biology, vol. 72, no. 5, pp. 169195, 2000.

[2] N. Doukyu, "Characteristics and biotechnological applications of microbial cholesterol oxidases," Applied Microbiology and Biotechnology, vol. 83, no. 5, pp. 825-837, 2009.

[3] H.-J. Cho, K.-P. Choi, M. Yamashita, H. Morikawa, and Y. Murooka, "Introduction and expression of the Streptomyces cholesterol oxidase gene (ChoA), a potent insecticidal protein active against boll weevil larvae, into tobacco cells," Applied Microbiology and Biotechnology, vol. 44, no. 1-2, pp. 133-138, 1995.

[4] S. Serajzadeh and I. Alemzadeh, "Low-cholesterol milk production using cholesterol oxidase from Nocardia erythropolis," Australian Journal of Dairy Technology, vol. 65, pp. 10-14, 2010.

[5] S. Devi and S. S. Kanwar, "Cholesterol oxidase: source, properties and applications," Insights in Enzyme Research, vol. 1, pp. 1-12, 2017.

[6] A. Srivastava, V. Singh, and C. K. M. Tripathi, "Scale up and optimization of cholesterol oxidase production from Streptomyces rimosus MTCC 10792 in a 3-L bioreactor," Environmental Sustainability, vol. 1, no. 1, pp. 99-107, 2018.

[7] M. T. Yazdi, Z. T. Yazdi, G. Zarrini, A. Ghasemian, Z. Sepehrizadeh, and N. H. Olyaee, "Purification and characterization of extra-cellular cholesterol oxidase from Rhodococcus sp. PTCC 1633," Biotechnology (Faisalabad), vol. 7, no. 4, pp. 751-756, 2008.

[8] B. D. Bholay, D. J. Gadekar, S. K. Sawant, and S. M. Sonawane, "Bacterial extracellular cholesterol oxidase and its pharmaceutical perspectives," International Journal of Current Microbiology and Applied Science, vol. 2, pp. 19-28, 2013.

[9] A. Brzostek, B. Dziadek, A. Rumijowska-Galewicz, J. Pawelczyk, and J. Dziadek, "Cholesterol oxidase is required for virulence of Mycobacterium tuberculosis," FEMS Microbiology Letters, vol. 275, no. 1, pp. 106-112, 2007.

[10] E. A. Elsayed, E. N. Danial, M. A. Wadaan, and H. A. ElEnshasy, "Production of $\beta$-galactosidase in shake-flask and 
stirred tank bioreactor cultivations by a newly isolated Bacillus licheniformis strain," Biocatalysis and Agricultural Biotechnology, vol. 20, p. 9, 2019.

[11] H. E. Lashgarian, S. Jahanbakhsh, and K. Shahzamani, "Molecular identification of cholesterol oxidase enzymeproducing Streptomyces bacteria in soil of Lorestan province," Iranian International Journal of Medical Research and Health Sciences, vol. 5, pp. 54-62, 2016.

[12] D. Wang, C. Wang, P. Gui et al., "Identification, bioactivity, and productivity of actinomycins from the marine-derived Streptomyces heliomycini," Frontiers in Microbiology, vol. 8, p. 12, 2017.

[13] N. Nadia, Z. A. Nehad, E. A. Elsayed, M. A. Essam, and M. A. Hanan, "Optimization of lipase synthesis by Mucor racemosus-production in a triple impeller bioreactor," Malaysian Journal of Microbiology, vol. 6, pp. 7-15, 2010.

[14] A. Z. Boumehira, H. A. El-Enshasy, H. Hacène, E. A. Elsayed, and R. Park, "Recent progress on the development of antibiotics from the genus Micromonospora," Biotechnology and Bioprocess Engineering, vol. 21, no. 2, pp. 199-223, 2016.

[15] E. A. Elsayed, M. A. Farid, and H. A. El-Enshasy, "Enhanced natamycin production by Streptomyces natalensis in shakeflasks and stirred tank bioreactor under batch and fed-batch conditions," BMC Biotechnology, vol. 19, p. 13, 2019.

[16] A. N. Kepli, D. J. Dailin, R. A. Malek, E. A. Elsayed, O. M. Leng, and H. A. El-Enshasy, "Medium optimization using response surface methodology for high cell mass production of Lactobacillus acidophilus," Journal of Scientific and Industrial Research, vol. 78, pp. 608-614, 2019.

[17] C. Then, O. K. Wai, E. A. Elsayed et al., "Comparison between classical and statistical medium optimization approaches for high cell mass production of Azotobacter vinelandii," Journal of Scientific and Industrial Research, vol. 75, pp. 231-238, 2016.

[18] K. R. A. Hamid, E. A. Elsayed, H. A. El-Enshasy, M. Esawy, and R. A. Malek, "Bioprocess optimization for levan production by Bacillus subtilis B58," Journal of Scientific and Industrial Research, vol. 77, pp. 386-393, 2018.

[19] M Soltani, R. Abd Malek, I. Ware et al., "Optimization of cordycepin extraction from Cordyceps militaris fermentation broth," Journal of Scientific and Industrial Research, vol. 76, pp. 355-361, 2017.

[20] F. Guo, X. Li, J. Zhao, G. Li, P. Gao, and X. Han, "Optimizing culture conditions by statistical approach to enhance production of pectinase from Bacillus sp. Y1," BioMed Research International, vol. 2019, Article ID 8146948, 10 pages, 2019.

[21] H. A. El Enshasy, E. A. Elsayed, N. Suhaimi, R. A. Malek, and M. Esawy, "Bioprocess optimization for pectinase production using Aspergillus niger in a submerged cultivation system," BMC Biotechnology, vol. 18, p. 13, 2018.

[22] J. Sambrook, E. F. Fritsch, and T. Maniatis, "Molecular cloning: a laboratory manual, 2nd edn by J. Sambrook, E. F. Fritsch and T. Maniatis, cold spring harbor laboratory press, 1989. \$115.00 (3 vols; 1659 pages) ISBN 087969309 6," Trends in Biotechnology, vol. 9, 1991.

[23] U. Edwards, T. Rogall, H. Blöcker, M. Emde, and E. C. Böttger, "Isolation and direct complete nucleotide determination of entire genes. Characterization of a gene coding for 16S ribosomal RNA," Nucleic Acids Research, vol. 17, no. 19 , pp. 7843-7853, 1989.

[24] T. A. Hall, "A user-friendly biological sequence alignment editor and analysis program for Windows 95/98/NT," Nucleic Acids Symposium, vol. 41, pp. 95-98, 1999.

[25] R. Niwas, V. Singh, R. Singh, D. Tripathi, and C. K. M. Tripathi, "Production, purification and characterization of cholesterol oxidase from a newly isolated Streptomyces sp," World Journal of Microbiology and Biotechnology, vol. 29, no. 11, pp. 20772085, 2013.

[26] R. L. Plackett and J. P. Burman, "The design of optimum multifactorial experiments," Biometrika, vol. 33, no. 4, pp. 305-325, 1946.

[27] Y. Inouye, K. Taguchi, A. Fujii, K. Ishimaru, S. Nakamura, and R. Nomi, "Purification and characterization of extracellular 3.BETA.-hydroxysteroid oxidase produced by Streptoverticillium cholesterolicum," Chemical \& Pharmaceutical Bulletin, vol. 30, no. 3, pp. 951-958, 1982.

[28] V. A. Steingrube, R. W. Wilson, B. A. Brown et al., "Rapid identification of clinically significant species and taxa of aerobic actinomycetes, including Actinomadura, Gordona, Nocardia, Rhodococcus, Streptomyces, and Tsukamurella isolates, by DNA amplification and restriction endonuclease analysis," Journal of Clinical Microbiology, vol. 35, no. 4, pp. 817-822, 1997.

[29] S. Bhasin and H. A. Modi, "Optimization of fermentation medium for the production of glucose isomerase using Streptomyces sp. SB-P1," Biotechnology Research International, vol. 2012, Article ID 874152, 10 pages, 2012.

[30] M. T. Yazdi, M. Zahraei, K. Aghaepour, and N. Kamranpour, "Purification and partial characterization of a cholesterol oxidase from Streptomyces fradiae," Enzyme and Microbial Technology, vol. 28, no. 4-5, pp. 410-414, 2001.

[31] R. Varma and S. Nene, "Biosynthesis of cholesterol oxidase by Streptomyces lavendulae NCIM 2421," Enzyme and Microbial Technology, vol. 33, no. 2-3, pp. 286-291, 2003.

[32] S. Yang and H. Zhang, "Optimization of cholesterol oxidase production by Brevibacterium sp. employing response surface methodology," African Journal of Biotechnology, vol. 33, pp. 8316-8322, 2012.

[33] D. V. L. Zea, A. Mayerhoff, I. C. Roberto, and T. T. Franco, "Purification of xylose reduxtase from Candida mogii in aqueous two-phase systems," Biochemical Engineering Journal, vol. 18, pp. 217-223, 2004.

[34] J. M. B. Macedo, L. M. F. Gottschalk, and E. P. S. Bon, "Lignin peroxidase and protease production by Streptomyces viridosporus T7A in the presence of calcium carbonate," Twentieth Symposium on Biotechnology for Fuels and Chemicals, vol. 77-79, pp. 735-744, 1999.

[35] L. F. Dobson and D. G. O'Shea, "Antagonistic effect of divalent cations $\mathrm{Ca} 2+$ and $\mathrm{Mg} 2+$ on the morphological development of Streptomyces hygroscopicus var. geldanus," Applied Microbiology and Biotechnology, vol. 81, no. 1, pp. 119-126, 2008.

[36] Q.-S. Li, Q.-H. Fang, J.-Y. Zhu, and J.-J. Zhong, "Hyperproduction of L-glutamate oxidase in submerged fermentation of Streptomyces sp. N1 with culture $\mathrm{pH}$ control and calcium addition," Applied Biochemistry and Biotechnology, vol. 80, no. 2, pp. 97-106, 1999.

[37] A. K. Chauhan, S. A. Survase, J. Kishenkumar, and U. S. Annapure, "Medium optimization by orthogonal array and response surface methodology for cholesterol oxidase production by Streptomyces lavendulae NCIM 2499," The Journal of General and Applied Microbiology, vol. 55, no. 3, pp. 171-180, 2009.

[38] Z. Moradpour, A. Ghasemian, A. Safari, M. Mohkam, and Y. Ghasemi, "Isolation, molecular identification and statistical optimization of culture condition for a new extracellular cholesterol oxidase-producing strain using response surface methodology," Annals of Microbiology, vol. 63, Article ID 941, 2013. 
[39] V. Praveen, A. Srivastava, and C. K. M. Tripathi, "Purification and characterization of the enzyme cholesterol oxidase from a new isolate of Streptomyces sp," Applied Biochemistry and Biotechnology, vol. 165, no. 5-6, pp. 1414-1426, 2011. 